\title{
Implantación de la técnica del "ojal” o "buttonhole" en una unidad de hemodiálisis hospitalaria: satisfacción del paciente
}

\author{
Laura Baena Ruíz , Beatriz Martín González ${ }^{1}$, Alicia Marcos Ayuso² \\ ${ }^{1}$ DUE Unidad de diálisis y experto en técnicas de diálisis, ${ }^{2}$ DUE Unidad de cuidados intensivos. Hospital Universitario \\ del Henares. Madrid. España
}

\begin{abstract}
Sr. Director
Por los profesionales del campo de la Nefrología y la Diálisis es conocida la vital importancia de contar con un acceso vascular óptimo desde que en 1945 se realizase el primer tratamiento renal sustitutivo con hemodiálisis. Esto es debido a que la calidad del acceso vascular (AV) condiciona la eficiencia y efectividad de dicha técnica, así como la morbilidad y calidad de vida de los pacientes sometidos a esta terapia ${ }^{1}$.
\end{abstract}

Para que un AV sea considerado óptimo debe reunir varias características: permitir el abordaje seguro y continuado al sistema vascular, proporcionar flujos suficientes que permitan suministrar la dosis de diálisis requerida y carecer de complicaciones. Sin embargo, a pesar de que, en la actualidad, este tipo de AV no existe, el que más se acerca a estos requisitos es la fístula arteriovenosa interna (FAVI) ${ }^{1}$. Por ello, se considera el acceso vascular de elección en los pacientes con insuficiencia renal crónica terminal que requieren hemodiálisis.

La evidencia científica coincide en la importancia de los cuidados enfermeros efectivos de la FAVI y de una técnica de punción adecuada para conseguir el correcto desarrollo y permeabilidad, prolongar su supervivencia y minimizar las complicaciones relacionadas con la disfunción (estenosis, aneurismas, trombosis, infección...).

El correcto uso y mantenimiento de este acceso vascular (AV) va a ser la piedra angular relacionada con el aspecto técnico de la hemodiálisis en el que la enfermería puede incidir.

\section{Correspondencia: \\ Laura Baena Ruiz \\ C/. Mar del Norte, 4. Portal B, $1^{\circ} \mathrm{A}$ \\ 28821 Coslada. Madrid \\ E-mail: laura.baena@gmail.com}

Se ha comprobado que la técnica de punción de la FAVI es motivo de ansiedad y disconfort para el paciente $y$, secundariamente para el personal del enfermería ${ }^{2}$. Además de influir en la supervivencia de la FAVI, dado que las punciones repetidas acaban alterando la morfología y características del vaso sanguíne ${ }^{3}$, como ya enunció Kronung en 1984 con su análisis de las consecuencias de las múltiples punciones, y la mejor técnica para evitar su daño ${ }^{4}$, debido a la elongación de la pared frontal del vaso provocada por el desplazamiento del tejido y al posterior llenado con un trombo del hueco generado tras la retirada de la aguja.

En función de la técnica que haya sido utilizada, las consecuencias derivadas de la repetición de la punción variarán:

- Técnica de punción "en área": Se desarrollan zonas aneurismáticas sucediéndose de zonas estenóticas adyacentes al área puncionada que, según la Ley Hidrodinámica de Bernoulli, tenderán a progresar debido a la distribución de presiones y velocidad. Además, esta técnica no está exenta de generar espejismos de punciones óptimas dada la facilidad de punción, disminución del dolor durante la misma y elevación del flujo conseguidos por la dilatación, destrucción de elasticidad y consecuente debilitamiento de la pared vascular.

- Técnica de punción "en escalera": Se dilata mínima y equitativamente la longitud de la vena, no desarrollando aneurismas, debido a la distribución de las punciones a lo largo de toda la longitud del vaso lo cual no siempre es sencillo cuando no existe un trayecto venoso bien desarrollado, pudiendo generar mayor dolor durante la canulación y aumento de punciones infructuosas y repetidas.

- Técnica de punción "en ojal" o "buttonhole": Existe una ausencia de dilataciones y estenosis cuando la punción se realiza repetida y exactamente en el mismo lugar del vaso utilizando una 
única trayectoria a través del túnel subcutáneo creado y consolidado, como en 2005 demostraron el Dr. Toma, en Japón, con la sección de un vaso con ojal (de un paciente fallecido) ${ }^{5}$ y el enfermero Tony Goovaertsen, en Bélgica, con su informe ultrasonográfico sobre la afectación de los vasos en donde ha sido utilizada ésta técnica6.

No obstante, en la actualidad, la punción en área está siendo utilizada en el $61 \%$ de las canulaciones, opuestamente a lo ocurrido con la aplicación de la técnica del ojal cuyo porcentaje queda reducido a un $6 \%^{7}$ aunque el $90,4 \%$ asegura conocer la técnica "buttonhole"

A pesar de que ya desde la década de los setenta existen publicados los primeros artículos acerca de la técnica del ojal, que posteriormente en 1992 sería denominada "constant-site", firmados por Twardowski, y al contrario de lo que pasa en otros países europeos, en España la técnica "buttonhole" es poco considerada como opción y su uso apenas está extendido, pero tiene grandes beneficios a los que poder sacar partido.

Por ello, y tras el éxito conseguido tras dos años y medio desde la implantación de esta técnica en Hemodiálisis Domiciliaria $(H D D)^{9}$ y la satisfacción sentida a nivel bilateral (tanto desde el punto de vista de profesionales como de pacientes), se ha comenzado su utilización en la sala de hemodiálisis de crónicos de nuestro hospital a pacientes tratados tanto con hemodiálisis (HD) como con hemodiafiltración Online (HDF OL).

Nos planteamos como objetivo, evaluar los resultados de la implantación en la unidad de hemodiálisis de crónicos de nuestro hospital, de la técnica de punción en ojal o "buttonhole" como técnica de elección en todos los pacientes.

Hemos realizado para ello, un estudio analítico longitudinal que iniciamos en abril de 2012.

El tamaño de la muestra ha sido de 9 pacientes, 1 en HDD y 8 en HD en sala de crónicos, con un total de 21 túneles subcutáneos.

Incluimos a los pacientes con necesidad de autopunción orientados a HDD, y los pacientes en sala de crónicos con FAVI de difícil punción (multipunciones fallidas, sin posibilidad de variación de los lugares de punción, que padezcan miedo intenso o dolor importante durante la punción del AV) y todos aquellos pacientes que, previa información sobre la técnica y consentimiento informado, estuviesen interesados en la misma. Quedaron excluidos aquellos pacientes que recibían el tratamiento en otros centros durante períodos vacacionales y no quisieran $y / 0$ pudieran realizar su autopunción durante estos períodos, dado el posible desconocimiento de la técnica que pudiera existir por parte de los profesionales de dichos centros.

La creación de los túneles subcutáneos se llevo a cabo a lo largo de 5 sesiones, en las que puncionamos con aguja roma (excepto en la primera punción) y al final de las cuales colocamos los dispositivos de poliuretano tras la hemostasia, siempre llevando a cabo una doble asepsia, antes y después de la colocación del mismo, con la aplicación de clorhexidina acuosa.

Para ello, fue necesaria la planificación de las sesiones de manera consecutiva programando para su realización a la misma enfermera. Solamente en el caso de una paciente se realizó la técnica en sesiones alternas debido a cambios en la dinámica de la unidad.

Para evaluar la satisfacción de los pacientes, entregamos un cuestionario donde se evalúa previo a la realización de la técnica, la información recibida, el conocimiento de la misma por parte del profesional, la información y atención recibidas durante su realización, y posteriormente a la aplicación del procedimiento, la mejoría respecto a la técnica previa, las molestias causadas durante el desarrollo (programación de más sesiones de HD) y si volvería a realizarse la técnica una vez experimentada. Se evaluó también el nivel de dolor con la técnica de punción anterior y con la técnica del ojal. Todo ello, a través de escalas tipo EVA de 1 a 10 donde 1 es "nada adecuado/nada de mejoría" y 10 es "totalmente adecuado/mejoría absoluta".

Además registramos las siguientes variables para la valoración de los resultados de la implantación de la técnica: tiempo de hemostasia, variación de flujo de bomba e incidencia de casos de infección.

Paralelamente, y de manera subjetiva, se han valorado las complicaciones asociadas, incidencia de hematomas y extravasaciones, tamaño de los aneurismas, número de punciones fallidas y/o multipunción, gasto en material utilizado e implicación del paciente en su tratamiento y autocuidado.

Como resultados de la implantación de la técnica Buttonhole en nuestra unidad, hemos registrado:

Reducción del dolor asociado a la técnica de punción de un 4,7 a un 8,8 favorable a la técnica del ojal ( $p:<0,001$ ).

Respuesta media sobre la mejoría respecto a la técnica previa fue de 9,1 favorable a "buttonhole".

Compensación de las molestias causadas fue de 9,4 favorable a "buttonhole". 
Respuesta unánime con valoración de un 10 a si se realizarían nuevamente la técnica del ojal.

Reducción del tiempo de hemostasia de $20 \pm 11$ a $12 \pm 4$ minutos ( $p: 0,02$ ).

Aumento del flujo de bomba de $365 \pm 35$ a $373 \pm$ 27 (p: n. s.).

Ausencia de infecciones.

Ausencia de complicaciones observadas asociadas a la técnica durante el periodo de estudio.

Reducción del número de hematomas y extravasaciones, no existiendo ningún episodio durante el período de estudio.

Estabilización del tamaño de los aneurismas existentes.

Reducción del número de punciones fallidas.

Reducción del coste del material utilizado debido a la retirada del uso de celulosas hemostáticas como agentes coagulantes y a la disminución del número de agujas utilizadas.

Implicación activa del paciente en su tratamiento y autocuidado manifestado por la existencia de 3 pacientes que llevan a cabo su autopunción.

Subjetivamente no se apreciaron diferencias significativas entre la realización de la técnica en sesiones consecutivas y alternas.

De los 21 túneles creados, tres fueron fallidos por dificultar para puncionar con aguja roma, por lo que se creó nuevo túnel. Es decir, en tres pacientes se realizaron tres túneles.

Ante estos resultados, debemos tener presente que el uso de la técnica "buttonhole" es, hoy en día, controvertido. Son muchos los estudios en los que se refiere un importante incremento de la tasa de infección al usar la técnica "buttonhole" comparándola con las técnicas más habituales de punción ${ }^{10,11,12,13}$. Sin embargo, en pocos de estos artículos se hace referencia a la técnica de asepsia utilizada durante el procedimiento. Las recomendaciones generales para evitar las infecciones son el uso de mascarilla, la aplicación de asepsia cuidadosa y la aplicación de antibioterapia tópica ${ }^{14}$. En nuestro caso, se usa siempre doble mascarilla (en el paciente y en la enfermera), guantes estériles y técnica de doble asepsia con clorhexidina acuosa, pero no hemos utilizado la profilaxis antibiótica tópica. Por el momento, no hemos tenido ningún episodio de infección local ni generalizada, pero el tiempo de estudio es muy limitado.

En cuanto a la reducción del tiempo de hemostasia, lo hemos encontrado reflejado en contadas ocasiones ${ }^{15,16}$, siendo uno de los aspectos menos estudiados. En nuestro caso, la reducción del mismo, como se ha mencionado antes, es uno de los principales beneficios que hemos ob- tenido, siendo estadísticamente significativo $(p=0.02)$.

La utilización del dispositivo de poliuretano para la creación del túnel es recomendable bajo nuestro punto de vista, ya que facilita que no se creen túneles alternativos que dificultan la punción con aguja roma. En la bibliografía vemos recogido que algunos estudios reflejan menor dolor con la utilización del dispositivo ${ }^{17}$. También se usa, en ocasiones, un catéter para estabilizar el túnel, pero esta modalidad ha dado más problemas que la anterior a lo largo de su utilización ${ }^{18,19}$.

Una de las diferencias también reseñables con bibliografía previa encontrada al respecto es el uso de agujas romas desde la segunda punción en lugar de comenzar su uso una vez que el túnel ya está estabilizado. De esta manera, evitamos la creación involuntaria de túneles adyacentes al originario ${ }^{14}$.

Desde el punto de vista del paciente, lo más beneficioso es la reducción del dolor. En nuestro estudio, hemos logrado una reducción muy significativa $(p:<0,001)$. La bibliografía refleja diferencias en cuanto al dolor según sea la técnica de realización del túnel con dispositivo de poliuretano 0 sin él ${ }^{20}$.

En lo referente a cargas de trabajo y dificultad para la aplicación de la técnica, es cierto que debe existir una implicación importante del personal de enfermería, ya que requiere un período de aprendizaje y cierto grado de disponibilidad para coordinar que las primeras punciones las realice la misma enfermera. Para ello, es imprescindible la colaboración de los supervisores de unidad, que deben facilitar a la enfermería los cambios de turno y rotaciones de libranzas para poder llevarlo a cabo. En nuestro caso, esto no ha sido de gran dificultad debido a la motivación de todo el personal de la unidad para implantar la nueva técnica.

Aún se necesita más experiencia y estudios más amplios para valorar el uso generalizado de la técnica "buttonhole" en el medio hospitalario. Aunque todos los autores coinciden en que es la técnica de elección para los pacientes en programa de hemodiálisis domiciliaria, su uso en las salas de crónicos en hospitales no está extendida aún.

Nuestra experiencia es corta para valorar parámetros como la tasa de infección, la supervivencia del acceso vascular o la formación de aneurismas, pero lo que sí hemos logrado es una reducción significativa del tiempo de hemostasia y del dolor relacionado con la punción valorada por los propios pacientes. Otro aspecto positivo es que algunos de los pacientes han comenzado a realizarse autopunción, con la consiguiente implicación en su tratamiento y, sobre todo, en el cuidado de su fístula arteriovenosa. 
Para nosotras, la valoración subjetiva de los pacientes cobra gran importancia, ya que son los destinatarios de nuestros cuidados $y$, por tanto, todo esfuerzo por parte del personal de enfermería nefrológica debe ir encaminado a mejorar el tratamiento, la tolerancia del mismo y la calidad de vida de nuestros pacientes. Por ello, incluimos la encuesta de satisfacción para los pacientes a los que se les ha realizado la técnica, ya que su opinión es crucial para valorar si la técnica del ojal es bien tolerada.

Desde nuestra experiencia, los resultados hasta ahora han sido positivos, pero vemos necesaria la ampliación del estudio incluyendo nuevos pacientes en la técnica y realizando un seguimiento más a largo plazo.

\section{Bibliografía}

1. Fernández Medina, IM: Cuidados de la fístula arteriovenosa durante la sesión de hemodiálisis. ENE. Revista de Enfermería. Abril 2013; 7 (I).

2. Grau Pueyo, $C$ et al: La punción del acceso vascular en hemodiálisis es una necesidad, el método Buttonhole una opción. Rev Soc Esp Enferm Nefrol. 2011; 14 (1): 30/36.

3. Pisoni, RL et al: Facility hemodialysis vascular Access use and mortality in countries participating in DOPPS: an instrumental variable analysis. Am J Kidney Dis. 2009; 53 (3): 475-91.

4. Hernando, L: Nefrología Clínica. $3^{a}$ Edición. Ed. Médica Panamericana. 2008; 19 (1). 889.

5. Toma, ST: Una técnica incorporando clavija de polipropileno para crear una ruta de punción fija para la técnica del ojal. Conferencia anual de diálisis. 2005. Florida.

6. Goovaerts, T: Experiencia a largo plazo con la técnica del ojal en la canalización de la fístula. Conferencia anual de diálisis. 2005. Florida.

7. Parisoto, MT: Estudio multicéntrico sobre la práctica de canulación a pacientes en hemodiálisis. Centros Fresenius Medical Care. 35 Congreso SEDEN. 2010. España.

8. González Molina, J et al: Análisis enfermero de la penetración de la técnica del ojal en las unidades de hemodiálisis españolas. Enfermería Nefrológica. 2012; 15 Suppl (1): 17/83.
9. Baena Ruiz, L: Buttonhole: técnica de punción de elección en hemodiálisis domiciliaria. Enferm Nefrol. 2014; 17 (2): 147/149.

10. Marticorena R, Hunter J, Macleod S, et al. The salvage of aneurysmal fistula utilizing a modified buttonhole cannulation technique and multiple cannulators. Hemodial Int 2006; 10:193-200.

11. Verhallen AM, Kooistra MP, van Jaarsveld BC. Cannulating in haemodialysis: rope-ladder or buttonhole technique? Nephrol Dial Transplant 2007; 22:2601-2604.

12. $0^{\prime}$ Brien FJ, Kok HKT, $0^{\prime}$ Kane $C$, et al. Arteriovenous fistula buttonhole cannulation technique: a retrospective analysis of infectious complications. Clin Kidney J 2012; 5:526-529.

13. Chow J, Rayment G, Miguel SS, Gilbert M. A randomised controlled trial of buttonhole cannulation for the prevention of fistula access complications. J Ren Care 2011; 37:85-93.

14. Rajneet K. Atkara, and Jennifer M. MacRae. The buttonhole technique for fistula cannulation: pros and cons. Mc Rae Current Opinion 2013.

15. Jennifer M. MacRae, Sofia B. Ahmed, ${ }^{\star}$ Rajneet Atkar, and Brenda R. Hemmelgarn. A Randomized Trial Comparing Buttonhole with Rope Ladder Needling in Conventional Hemodialysis Patients. October, 2012. Clin J Am Soc Nephrol 7: 16321638.

16. Kim MK, Kim HS. Clinical effects of buttonhole cannulation method on hemodialysis patients. Hemodial Int 2013; 17:294-299.

17. Marticorena RM, Hunter J, Mc Leod S, et al. Use of the BioHole device for the creation of tunel tracks for buttonhole cannulation of fistula for hemodialysis. Hemodial int. 2011. 15:243-249.

18. Donnelly SM, Marticorena RM, Hunter J, Goldstein MB. Supercath safety Clampcath buttonhole creation: complication of catheter breakage. Hemodial Int 2013; 17 (3):450-454.

19. Macrae JM, Tai DJ, Daniw M, Lee J. A simple method to create buttonhole cannulation tracks in a busy hemodialysis unit. Hemodial Int 2010; 14:94-95.

20. Toma $S$, Shinzato T, Fukui $H$, et al. A timesaving method to create a fixed puncture route for the buttonhole technique. Nephrol Dial Transplant 2003; 18:2118-2121. 